\title{
Traducão e validação psicométrica do Behavioral Reǵulation in Exercise Questionnaire para uso em adultos brasileiros
}

\section{Translation and psicometric validation of the Behavioral Regulation in Exercise Questionnaire for use in brazilian adults}

Dartagnan Pinto Guedes ${ }^{1}$

Sandro Lucas Sofiati ${ }^{1}$

\section{RESUMO}

Os objetivos do estudo foram traduzir para o idioma português, realizar a adaptação transcultural e identificar as propriedades psicométricas do Behavioral Regulation in Exercise Questionnaire - Version 3 (BREQ-3) para uso em adultos brasileiros. O questionário original foi traduzido de acordo com recomendações internacionais. Um comitê de juízes foi formado para analisar as versões traduzidas do questionário. O comitê utilizou como critério de análise as equivalências semântica, idiomática, cultural e conceitual. A versão final do questionário traduzido foi administrada em uma amostra de 1041 adultos (55\% homens) praticantes de exercício físico da área metropolitana da cidade de Londrina, Paraná com idades $\geq 18$ anos. Para identificar as propriedades psicométricas iniciais foi realizada análise fatorial exploratória e, na sequência, análise fatorial confirmatória. Para análise da consistência interna de cada fator associado à motivação para a prática de exercício físico foi empregado coeficiente alfa de Cronbach. Após discretas modificações apontadas no processo de tradução, o comitê de juízes considerou que a versão para o idioma português do $B R E Q-3$ apresentou equivalências semântica, idiomática, cultural e conceitual. A análise fatorial confirmou a estrutura de seis fatores originalmente proposta, mediante indicadores estatísticos equivalentes à $\chi^{2} / \mathrm{gl}=1,91$, ao GFI $=0,938, \mathrm{AGFI}=0,954$ e $\mathrm{RMSR}=0,059$ $\left(\mathrm{IC}_{95 \%}\right.$ 0,051-0,069). O alfa de Cronbach apresentou coeficientes entre 0,67 e 0,87. Concluindo, a tradução, a adaptação transcultural e as qualidades psicométricas do BREQ-3 foram satisfatórias, o que viabiliza sua aplicação em futuros estudos no Brasil.

\section{PALAVRAS-CHAVE}

Questionários; Psicometria; Exercício físico; Motivação; Brasil.

\begin{abstract}
The objectives of this study were to translate for the Portuguese language, describe the cross-cultural adaptation and identify the psychometric properties of the Behavioral Regulation in Exercise Questionnaire - Version 3 (BRSQ-3) for use in Brazilian adults. The original questionnaire was translated following international recommendations. Translated versions of the questionnaire were analyzes by a committee of experts. The committee used semantic, idiomatic, cultural and conceptual equivalences as criteria of analysis. The final version of the translated questionnaire was administered in a sample of 1041 adults (55\% men) exercise participants aged $\geq 18$ years-old. To identify initial psychometric properties exploratory factorial analysis were completed and, sequentially confirmatory factorial analysis. Cronbach's alpha coefficient was used to assess the internal consistency of each factor of the BREQ-3 associated to motivation for the practice of exercise. After minor changes identified in the translation process, the committee of experts considered that the Portuguese version of the BREQ3 showed semantic, idiomatic, cultural and conceptual equivalences. The factorial analysis confirmed the structure of six factors originally proposed, through statistical indicators equivalent to $\chi^{2} / g l=$ $1,91, G F I=0,938, A G F I=0,954$ e RMSR =0,059 $\left(I C_{95 \%}\right.$ 0,051-0,069). The Cronbach-alpha ranged from 0,67 to 0,87. In conclusion, the translation, cross-cultural adaptation and psychometric qualities of the BREQ-3 were satisfactory, thus enabling its application in future studies in Brazil.
\end{abstract}

\section{KEYWORDS}

Questionnaires; Psychometrics; Exercise; Motivation; Brazil.
Rev Bras Ativ Fís Saúde p. 397-412 DOI

http://dx.doi.org/10.12820/rbafs.v.20n4p397

1 Universidade Norte do Paraná, Centro de Pesquisa em Ciências da Saúde, Londrina, Paraná, Brasil 
Os múltiplos benefícios que a prática adequada de exercício físico possa oferecer à promoção do bem-estar e à minimização dos riscos predisponentes ao aparecimento e ao desenvolvimento de disfunções degenerativas são bem documentados na literatura ${ }^{1}$. Porém, contraditoriamente, levantamentos epidemiológicos têm apontado que pequena parcela da população pratica exercício físico com alguma regularidade ${ }^{2,3} \mathrm{e}$, entre aqueles que iniciam a sua pratica, a elevada quantidade de casos de abandono torna-se bastante preocupante ${ }^{4,5}$. Neste sentido, tem-se apontado o perfil motivacional individual como elemento decisivo para identificação e adesão a prática de exercício físico ${ }^{6}$.

Diante desta situação, estudos mais recentes vêm procurando se utilizar de pressupostos assumidos por diferentes abordagens teóricas elaboradas no domínio da psicologia para demarcar e conhecer atributos vinculados aos componentes motivacionais que possam levar alguém a praticar exercício físico ${ }^{6}$. A identificação de atributos associados à motivação subjacentes ao exercício físico permite que sejam delineadas ações de incentivo para o início de sua prática e que possam levar o praticante a alcançar em sua maior plenitude as metas propostas. Desse modo, tende a promover um clima motivacional favorável, o que aumenta as chances de adesão e, por consequência, minimiza eventual possibilidade de abandono.

Mais recentemente, sobretudo nas duas últimas décadas, a Teoria de Autodeterminação $(T a D)$ tem se constituído na abordagem teórica de maior relevância e que vem recebendo considerável atenção para estudo da motivação voltada à prática de exercício físico ${ }^{7,8}$. A TaD é uma teoria geral do comportamento humano que tem conexão com a personalidade individual e o contexto social em que se esta inserido. Neste sentido, procura explicar a motivação em diversas áreas, como educação, música, saúde, política, esporte e exercício físico, entre outras. Em síntese, postula que a motivação para assumir condutas específicas pode variar ao longo de um continuum de acordo com o grau percebido de autodeterminação, estando alicerçado nas três necessidades psicológicas básicas - (a) autonomia: tomar decisões, ter liberdade de escolha; (b) competência: sentir-se eficaz e capaz de alcançar os objetivos; e (c) relacionamento: manter uma relação amistosa com as pessoas ${ }^{9}$.

Em um extremo do continuum de autodeterminação está situado o construto denominado amotivação, que implica na ausência de qualquer tipo de motivação (intrínseca ou extrínseca) para a prática de exercício físico. A amotivação supõe o menor grau de autodeterminação, considerando que, neste caso, o indivíduo não encontra nenhum valor ou sentido na atividade que realiza e, portanto, considera que o exercício físico não reportará satisfação ou qualquer tipo de benefício. No outro extremo do continuum se encontra a motivação intrínseca. Este tipo de motivação supõe a forma mais autodeterminada de motivação, considerando que o indivíduo pratica exercício físico por vontade própria, ou seja, pelo prazer e satisfação que a atividade oferece a ele. Entre os dois polos do continuum (amotivação e motivação intrínseca) encontra-se a motivação extrínseca - figura 1 .

A $T a D$ distingue quatro tipos de regulação dentro da motivação extrínseca, que podem variar grandemente dependendo do grau de internalização das metas e dos valores associados a conduta de exercício físico: regulação externa: não exis- 


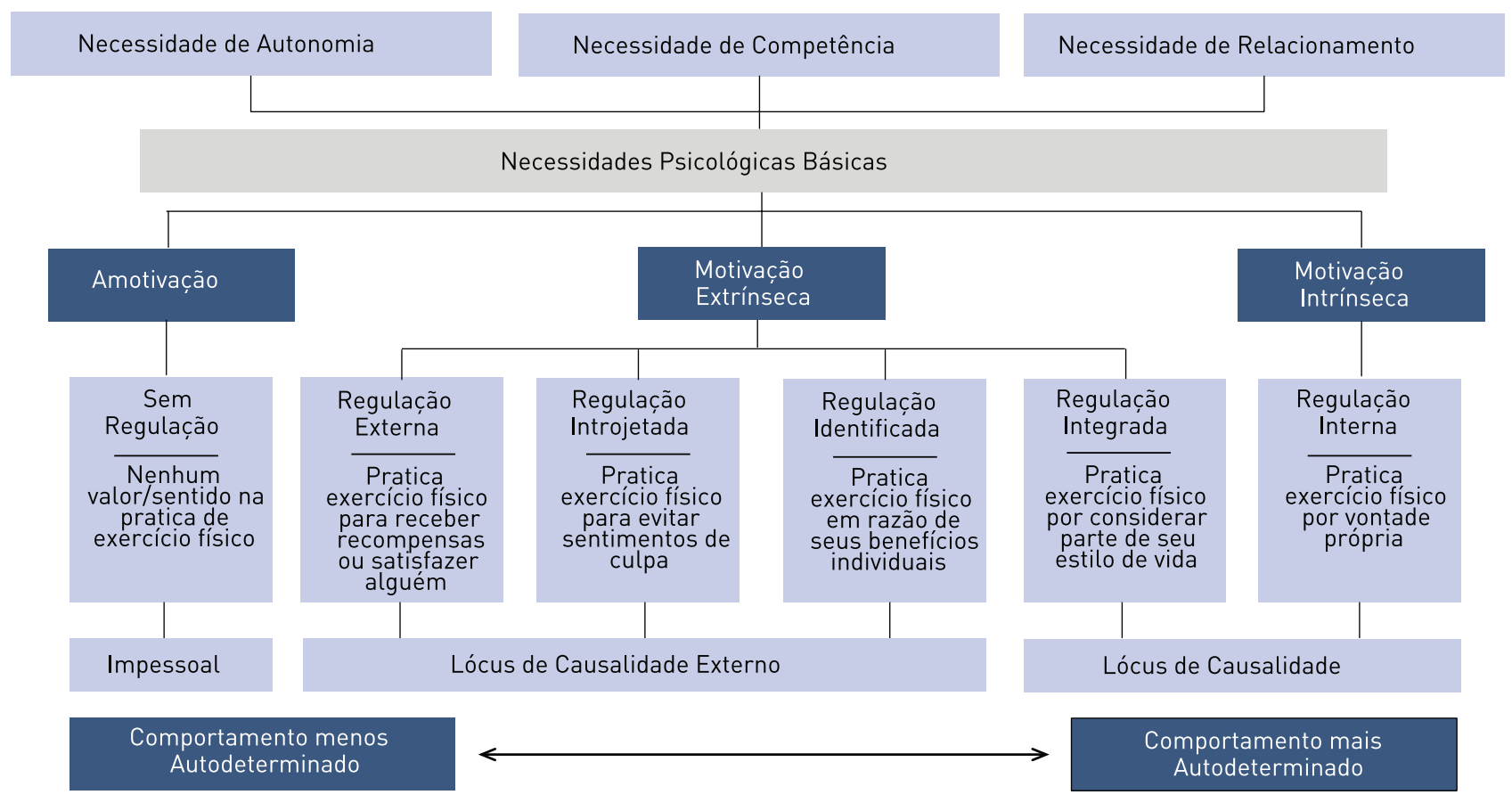

FIGURA 1 - Esquema geral da Teoria da Autodeterminação adaptada para a prática de exercício físico.

te internalização e o exercício físico é praticado unicamente por conta de recompensas externas ou para satisfazer alguém de seu convívio; regulação introjetada: surge um mínimo de internalização e o exercício físico é praticado para evitar sentimentos de culpabilidade; regulação identificada: apresenta grau de internalização mais elevado quanto ao valor da conduta, de tal modo que o exercício físico é praticado em razão da importância e dos benefícios individuais que sua prática pode propiciar; e regulação integrada: existe maior grau de internalização e a prática de exercício físico é considerada como parte do seu estilo de vida.

Estudos experimentais têm mostrado que indivíduos com perfis motivacionais direcionados à regulação identificada, à regulação integrada e à motivação intrínseca tendem a apresentar atitudes mais favoráveis para a prática de exercício físico, mediante mais elevada autoestima, satisfação, esforço e persistência; enquanto indivíduos com perfis motivacionais direcionados à regulação introjetada, à regulação externa e à amotivação tendem a apresentar processos adaptativos para a prática de exercício físico menos favoráveis, aumentando as chances de abandono, ${ }^{9,10}$.

Se, por um lado, os fundamentos da $T a D$, por serem baseados em características inerentes ao ser humano, são possíveis de generalização para diferentes culturas ${ }^{11}$; por outro, existe necessidade de dispor de instrumentos de medida traduzidos e validados para diferentes culturas capazes de identificar e dimensionar os construtos associados ao continuum de autodeterminação. Neste particular, no panorama internacional, encontram-se disponíveis na literatura dois questionários idealizados especificamente para atender o contexto de exercício físico: Exercise Motivation Scale -EMS ${ }^{12}$ e Behavioral Regulation in Exercise Questionnaire - BREQ ${ }^{13-15}$.

A proposição do $E M S$ recebeu severas críticas, entre outros motivos, por não proporcionar apoio psicométrico específico para os itens da regulação integrada, caracterizada como a forma mais autônoma de motivação extrín- 
seca prevista na TaD. Ainda, análise das correlações inter-construto apontou que as diferentes formas de motivação extrínseca não se associavam conforme postulado pela $T a D$, tendo sido encontrada correlação mais elevada entre a regulação integrada e a regulação introjetada, que entre a regulação integrada e a regulação identificada. Em vista disso, o $E M S$ vem passando despercebido pelos estudiosos da área, sendo dispensada maior atenção ao uso do $B R E Q^{16}$.

O BREQ foi originalmente idealizado por Mullan et al. ${ }^{13}$ e, na sequência, foi revisado e complementado mediante duas outras versões. A primeira versão do $B R E Q$ foi proposta para oferecer indicações vinculadas à motivação intrínseca e a três regulações da motivação extrínseca: regulação externa, introjetada e identificada. Apesar dos esforços de seus idealizadores, na sequência, reconheceu-se importante limitação da versão original, em razão da ausência de itens relacionados à amotivação. Neste sentido, foi disponibilizada versão ajustada do $B R E Q$, o que se denominou $B R E Q-2$, mantendo os quatro fatores da estrutura original da escala; porém, adicionando quatro novos itens equivalentes à amotivação ${ }^{14}$. A versão mais recente do $B R E Q$, o chamado $B R E Q-3$, foi sugerida com intuito de adicionar itens direcionados a atender a regulação integrada, passando, desse modo, a contemplar todos os seis construtos do continuum de autodeterminação ${ }^{15}$.

Todas as três versões originais do $B R E Q$ foram propostas em língua inglesa; no entanto, pesquisadores de países de outros idiomas se interessaram pela sua tradução e validação, o que vem permitindo a expansão de seu uso para outras culturas ${ }^{17-20}$. No momento, tem-se constituído em um dos instrumentos mais utilizado na literatura internacional sobre a TaD relacionada ao contexto de exercício físico ${ }^{21-24}$.

Especificamente em idioma português foram identificadas duas tentativas de tradução e validação do $B R E Q$. Uma primeira tentativa envolveu o idioma português europeu e contemplou o $B R E Q-2^{18}$. Neste caso, foram mantidos na versão traduzida os 19 itens propostos inicialmente, com disposição fatorial similar a encontrada originalmente e com aceitáveis indicadores de validação psicométrica. Contudo, pelo fato do $B R E Q$ tratar-se de um instrumento que contém extensa lista de diferentes razões para a prática de exercício físico expressas em frases, sua tradução para outro idioma, mesmo um idioma bastante similar ao utilizado no Brasil como é o caso do português europeu, pode comportar divergências em expressões idiomáticas e de matrizes provenientes de diferenças culturais. Assim, sua utilização na realidade brasileira pode levar a viés idiomático importante.

Tentativa de adaptar o $B R E Q$ para uso no Brasil foi realizada por Via$\mathrm{na}^{25}$. Contudo, neste caso, foi utilizado como referência a versão já traduzida para o idioma português europeu do $B R E Q-2$, ao invés de recorrer a versão do instrumento no seu idioma original (inglês). Desse modo, no delineamento do estudo são identificadas importantes limitações metodológicas associadas à tradução e à adaptação transcultural de questionários com essas características, o que pode comprometer sua aplicação na população brasileira. Somado a isso, a amostra envolvida no estudo para identificar os indicadores psicométricos foi constituída por 87 adolescentes, com idade média de 16 anos, ou seja, quantidade insuficiente de sujeitos para que se possa alcançar ajuste do modelo fatorial estatisticamente adequado com 19 itens ${ }^{26}$. Por consequência, sólidos critérios conceituais cederam preferência aos comprometidos achados estatísticos.

Em sendo assim, diante da necessidade de disponibilizar instrumento de 
medida apropriado à cultura brasileira que possa ser empregado para identificar, dimensionar e ordenar os tipos de motivação voltados à prática de exercício físico à luz da $T a D$, os objetivos do estudo foram traduzir para o idioma português, realizar a adaptação transcultural e identificar as propriedades psicométricas do $B R E Q-3$ para uso em adultos brasileiros.

\section{MÉTODOS}

\section{Instrumento}

A versão $B R S Q-3$ foi concebida com 23 itens, precedidos pelo enunciado "Why do you engage in exercise?/Porque você pratica exercício físico?", em que o respondente indica o grau de concordância que mais se aplica ao seu caso, por intermédio de uma escala de medida tipo Likert de cinco pontos $(0=$ "Not true for me/Nada verdadeiro para mim"; 2 = "Sometimes true for me/Algumas vezes verdadeiro para mim"; 4 = "Very true for me/Muito verdadeiro para mim"). $\mathrm{Na}$ sequência, mediante tratamento dos escores atribuídos a cada item e baseando-se no continuum de autodeterminação, torna-se possível identificar, dimensionar e ordenar seis escalas de motivação: (a) amotivação - AMOT; (b) motivação extrínseca de regulação externa - REEX; (c) motivação extrínseca de regulação introjetada - REIJ; (d) motivação extrínseca de regulação identificada - REID; (e) motivação extrínseca de regulação integrada - REIG; e (f) motivação intrínseca - MOTI $^{15}$.

\section{Tradução e Adaptação Transcultural}

Os protocolos de tradução e adaptação transcultural acompanharam procedimentos sugeridos internacionalmente ${ }^{27}$. A tradução inicial do idioma original (inglês) para o português foi realizada de maneira independente por dois pesquisadores com entendimento detalhado do $B R E Q$. Os dois pesquisadores tinham como idioma nativo o português e amplo domínio do idioma inglês, com experiência em traduções de textos acadêmicos. Além da tradução, foi solicitado que registrassem expressões que poderiam oferecer dúbia interpretação.

Um grupo bilíngue formado por três pesquisadores da área de exercício físico comparou os textos traduzidos, uniformizando o uso de expressões divergentes, e foi produzida uma versão única do questionário que sintetizou as duas versões anteriores. Em seguida, ocorreu a retrotradução do questionário por dois outros tradutores de maneira independente. Os tradutores escolhidos para essa etapa tinham como idioma nativo o inglês, domínio do idioma português e atuação como docente universitário em Instituição brasileira. Solicitou-se aos tradutores que registrassem expressões que pudessem gerar dúvidas no processo de retrotradução. $\mathrm{O}$ grupo bilíngue comparou ambos os textos retrotraduzidos, produzindo versão única.

Um comitê analisou o processo de tradução e os resultados alcançados nas etapas anteriores. O comitê foi formado por nove membros, incluindo os autores do estudo, tradutores que participaram do processo de tradução/ retrodução e três docentes universitários da área do esporte, todos bilíngue inglês-português. O comitê realizou revisão das sete versões do $B R E Q$ disponível: versão original em língua inglesa, duas versões traduzidas para o idioma português, versão síntese de ambas as traduções para o idioma português, duas versões de retrotradução e versão síntese de ambas as retrotraduções. 
O comitê realizou apreciação dos tipos de equivalências entre o instrumento original e a versão no idioma português. Os membros receberam orientações por escrito sobre o objetivo do estudo e as definições adotadas para as equivalências. Cada um respondeu individualmente a um formulário de análise que comparava cada item do questionário original, da versão síntese traduzida para o idioma português e da versão síntese de retrotradução, em relação às equivalências semântica, idiomática, cultural e conceitual. $\mathrm{O}$ formulário de análise foi estruturado mediante escala diferencial com alternativas discretas: "inalterada", "pouco alterada", "muito alterada" e "completamente alterada".

\section{Sujeitos}

Próxima etapa do estudo foi realizar testagem do $B R E Q-3$ traduzido para o português, com intuito de identificar os indicadores de validade psicométrica. Para tanto, o BREQ-3 foi aplicado em uma amostra de adultos frequentadores de Clube de Fitness da cidade de Londrina, Paraná. Para seleção dos sujeitos para compor a amostra, em vista da impossibilidade de utilizar procedimento de aleatoriedade simples, e considerando-se a dificuldade de relacionar todo universo da população tratada, optou-se por visitar todos os Clubes de Fitness identificados na cidade de Londrina por três vezes, em dias da semana e em horários diferentes, e convidar para participarem voluntariamente do estudo aqueles sujeitos que eventualmente foram localizados. Após serem informados sobre o estudo e concordarem em participar, assinaram o Termo de Consentimento Livre e Esclarecido, e receberam o instrumento de medida autoaplicável e anônimo para preenchimento. Neste caso, a amostra foi constituída por 1041 sujeitos (470 mulheres e 571 homens), com idades entre 18 e 60 anos.

\section{Procedimentos}

Para caracterização da amostra envolvida no estudo, adicionalmente aos itens do $B R E Q-3$, foram levantadas informações quanto ao sexo, à idade, ao nível econômico e ao histórico de prática de exercício físico: modalidade praticada, frequência semanal, duração das seções, longevidade de pratica e principal objetivo com a prática de exercício físico. Para identificar a classe econômica recorreu-se a diretriz proposta pela Associação Nacional de Empresas de Pesquisa ${ }^{28}$.

A coleta de dados foi realizada entre os meses de fevereiro e julho de 2014 por um único pesquisador conhecedor do instrumento e treinado quanto aos procedimentos. A aplicação do instrumento de medida ocorreu no próprio local de realização de exercício físico do sujeito, sendo mantidos em todos os casos os mesmos critérios e condições de aplicação. Por vezes, para não prejudicar a seção de exercício físico do sujeito selecionado para o estudo, foram agendados dia e horário específicos para retorno do pesquisador e aplicação do instrumento.

Os participantes do estudo receberam o instrumento de medida com instruções e recomendações para autopreenchimento, não sendo estabelecido limite de tempo para o seu término. $\mathrm{O}$ instrumento de medida foi respondido individualmente, sem qualquer contato com outras pessoas. Eventuais dúvidas manifestadas pelos respondentes foram prontamente esclarecidas pelo pesquisador que estava acompanhando a coleta de dados. Após o seu preenchimento, o instrumento de medida foi armazenado pelo respondente em uma urna juntamente com todos os demais, garantindo-se, dessa forma, o anonimato. As aplicações dos instrumentos de medida tiveram duração de 
15 a 30 minutos. O estudo foi aprovado pelo Comitê de Ética em Pesquisa Envolvendo Seres Humanos da Universidade Norte do Paraná - Plataforma Brasil (Parecer 208.975/2013).

\section{Tratamento Estatístico}

Inicialmente, envolvendo toda a amostra selecionada, foram calculados valores de média e desvio-padrão, acompanhados das indicações de simetria e curtose de cada item do inventário para verificar o comportamento de normalidade univariada da distribuição. A normalidade multivariada foi analisada mediante coeficiente de Mardia assumindo procedimento de bootstrapping. Na sequência, especificamente para identificar as propriedades psicométricas do BREQ-3, a amostra total foi dividida aleatoriamente em dois subconjuntos independentes de igual tamanho, assegurando a representatividade proporcional quanto à distribuição dos participantes nos estratos referentes ao sexo e à idade.

No primeiro subconjunto $\left(\mathrm{n}_{1}=520\right)$ foi empregada análise fatorial exploratória (AFE), por intermédio da técnica de componentes principais com rotação ortogonal (Varimax). A adequação do subconjunto de dados para os procedimentos da AFE foi verificada mediante testes estatísticos de KaiserMeyers-Olkin (KMO) e esfericidade de Bartlett. A matriz fatorial dos escores derivada dos 23 itens foi observada mediante análise da saturação fatorial itens-fatores. Para tanto, recorreu-se as correlações bivariadas por intermédio do coeficiente de correlação de Pearson. Neste caso, foi assumido como critério de exclusão aqueles itens com saturação fatorial inferior a 0,40 ou que estivessem representados em mais de um fator com saturação fatorial $\geq 0,40$. Para análise da consistência interna foram empregados os cálculos de alfa de Cronbach, acompanhados dos valores de média, desvio-padrão e correlações bivariadas inter-fatores.

Os procedimentos da análise fatorial confirmatória (AFC) por meio do método de estimativa Maximum Likelihood (máxima verossimilhança) foram conduzidos com os dados reunidos no segundo subconjunto da amostra $\left(\mathrm{n}_{2}\right.$ = 521) com intuito de identificar indicadores de validação equivalentes à estrutura fatorial extraída mediante a AFE. Quanto aos indicadores associados à validade de construto, o ajuste entre o modelo teórico proposto e a matriz de dados foi testado mediante múltiplos critérios: razão entre qui-quadrado e graus de liberdade $\left(\chi^{2} / \mathrm{gl}\right)$, Comparative Fit Index (CFI), Goodness-of-Fit Index (GFI), Adjusted Goodness-of-Fit Index (AGFI) e Root Mean Square Residual (RMSR). Neste caso, assumiu-se que $\chi^{2} / \mathrm{gl}<3$, CFI, GFI e AGFI $\geq 0,9$ juntos com valores de RMSR $\leq 0,08$ sugerem um bom ajuste do modelo ${ }^{29}$.

A validade convergente foi estimada por intermédio da variância extraída média (VEM) e da confiabilidade composta (CC), em que valores de VEM $\geq$ 0,50 e $\mathrm{CC} \geq 0,70$ foram considerados indicativos de validade adequada ${ }^{26}$. Adicionalmente, para estimar a invariância fatorial do modelo ajustado para uso do $B R E Q-3$ em praticantes de exercício físico de ambos os sexos e de diferentes idades foi conduzida análise multigrupo, fixando-se cargas fatoriais, variância/ covariâncias e resíduos. Para identificar eventuais diferenças significativas entre os submodelos extraídos separadamente por sexo (mulheres versus homens) e idade ( $\leq 20$ anos versus $21-40$ anos versus $\geq 41$ anos) foram consideradas diferenças entre valores de qui-quadrado $\left(\Delta \chi^{2}\right)$, respectivos graus de liberdade $(\Delta \mathrm{gl})$ e CFI $(\Delta \mathrm{CFI})$. Valores de $\mathrm{p}>0,05$ para $\Delta \chi^{2}$ e $\Delta \mathrm{CFI} \leq 0,01$ foram os 
critérios assumidos para definir invariância fatoria ${ }^{30}$. Os dados foram tratados utilizando-se os pacotes estatísticos computadorizados SPSSv20 e AMOSv20.

\section{Resultados}

Discretas divergências no uso de expressões foram observadas nas etapas do processo de tradução. As eventuais divergências foram discutidas no comitê de análise e prevaleceram as expressões de mais fácil compreensão e de uso frequente para facilitar o entendimento. Dos 23 itens da versão traduzida do BREQ-3, em 18 deles (78\%) os membros do comitê de análise apontaram como "inalterada" as equivalências semântica, idiomática, cultural e conceitual. Nos cinco restantes (22\%), os membros do comitê apontaram "pouco altera$d a$ " pelo menos em uma das equivalências. Nenhum item da versão traduzida do BREQ-3 apresentou as opções "muito alterada" ou "completamente alterada" assinaladas em comparação com a versão original.

Características da amostra selecionada para o estudo estão descritas na tabela 1. Dos 1041 sujeitos envolvidos no estudo, 54,9\% eram homens e, considerando ambos os sexos, 50,6\% deles tinham entre 21-30 anos e proporção similar se distribuiu em idades $\leq 21$ anos e $\geq 31$ anos. Com relação ao nível econômico, $67,1 \%$ dos sujeitos apresentaram informações equivalentes ao estrato econômico intermediário; contudo, maior proporção de mulheres $(21,7 \%)$ se localizou no estrato mais elevado economicamente e maior proporção de homens $(20,7 \%)$ no estrato mais baixo.

No que se refere ao histórico de prática de exercício físico, constatou-se predomínio de prática de exercícios resistidos; sobretudo nos homens (79,9\% versus $53,8 \%)$. No entanto, maior proporção de mulheres relatou praticar exercícios cardiorrespiratórios (26,2\%), pilates e outras modalidades alternativas de exercício físico (17,2\%). Quanto à frequência das seções de prática de exercício físico, identificou-se menor quantidade de seções/semana entre as mulheres, sendo que, $44,3 \%$ delas apontaram realizar exercício físico $\leq 5$ vezes/ semana em comparação com $61,5 \%$ dos homens. Com frequência equivalente a 3-4 vezes/semana observou-se 50,4\% das mulheres e 35,6\% dos homens. De maneira similar em ambos os sexos, menor proporção de sujeitos reunidos na amostra relataram se exercitar $\leq 60$ minutos/seção $(22,3 \%$ ), enquanto $32,6 \%$ apontaram se exercitar entre 60-90 minutos/seção. Quanto à longevidade de prática de exercício físico, identificou-se menor experiência entre as mulheres, sendo que, $41,1 \%$ delas apontaram ter $\leq 6$ meses de prática de exercício físico em comparação com $26,4 \%$ dos homens. Com prática de exercício físico $\geq 2$ anos observou-se $21,7 \%$ das mulheres e $40,1 \%$ dos homens. Se, entre as mulheres, estética $(36,8 \%)$ foi o principal objetivo a ser alcançado com a prática de exercício físico, no caso dos homens, prevaleceu condicionamento físico $(47,8 \%)$. Objetivos de saúde foram apontados igualmente em ambos os sexos por aproximadamente $1 / 4$ dos sujeitos reunidos no estudo.

Valores de média e desvio-padrão acompanhados dos índices de assimetria e curtose para cada item individualmente referente à amostra selecionada (n = 1041) são apresentados na Tabela 2. As pontuações atribuídas aos 23 itens inclusos no modelo, de maneira geral, apresentaram distribuição de dados normal (assimetria e curtose no intervalo \pm 1 ). Os índices mais elevados de assimetria e curtose foram obtidos no item 2 - Não sei por que tenho que fazer exercício físico (assimetria $=1,17$ e curtose $=1,21)$. Valores de média variaram de 
0,52 a 3,48, com desvios-padrão associados entre 0,51 e 1,25. A normalidade multivariada dos dados foi confirmada com índice de Mardia $=3,51$.

TABELA 1 - Características da amostra analisada no estudo.

\begin{tabular}{|c|c|c|c|}
\hline & $\begin{array}{l}\text { Mulheres } \\
(n=470)\end{array}$ & $\begin{array}{l}\text { Homens } \\
(n=571)\end{array}$ & $\begin{array}{c}\text { Ambos os Sexos } \\
(n=1041)\end{array}$ \\
\hline \multicolumn{4}{|l|}{ Idade } \\
\hline$\leqslant 20$ anos & $107(22,8 \%)$ & $137(24,0 \%)$ & $244(23,4 \%)$ \\
\hline $21-30$ anos & $232(49,4 \%)$ & $295(51,7 \%)$ & $527(50,6 \%)$ \\
\hline $31-40$ anos & $93(19,8 \%)$ & $95(16,7 \%)$ & $188(18,1 \%)$ \\
\hline$\geqslant 41$ anos & $38(8,2 \%)$ & $44(7,8 \%)$ & $82(8,0 \%)$ \\
\hline \multicolumn{4}{|l|}{ Nível Econômico } \\
\hline Elevado & $100(21,3 \%)$ & $71(12,5 \%)$ & $171(16,4 \%)$ \\
\hline Intermediário & $316(67,2 \%)$ & $382(66,9 \%)$ & $698(67,1 \%)$ \\
\hline Baixo & $54(11,5 \%)$ & $118(20,7 \%)$ & $172(16,5 \%)$ \\
\hline \multicolumn{4}{|l|}{ Modalidade de Exercício } \\
\hline Cardiorrespiratórios & $123(26,2 \%)$ & $82(14,4 \%)$ & $205(19,7 \%)$ \\
\hline Resistidos & $253(53,8 \%)$ & $456(79,9 \%)$ & $709(68,1 \%)$ \\
\hline Pilates/Alternativos & $81(17,2 \%)$ & $24(4,2 \%)$ & $105(10,1 \%)$ \\
\hline Aquáticos & $13(2,8 \%)$ & $9(1,6 \%)$ & $22(2,1 \%)$ \\
\hline \multicolumn{4}{|l|}{ Frequência } \\
\hline$\leqslant 2$ seções/semana & $25(5,3 \%)$ & $17(3,0 \%)$ & $42(4,1 \%)$ \\
\hline 3 - 4 seções/semana & $237(50,4 \%)$ & $203(35,6 \%)$ & $440(42,3 \%)$ \\
\hline$\geqslant 5$ seções/semana & $208(44,3 \%)$ & $351(61,5 \%)$ & $559(53,7 \%)$ \\
\hline \multicolumn{4}{|l|}{ Duração } \\
\hline$\leqslant 60$ minutos/seção & $104(22,1 \%)$ & $128(22,4 \%)$ & $232(22,3 \%)$ \\
\hline 60 - 90 minutos/seção & $216(46,0 \%)$ & $238(41,7 \%)$ & $454(43,6 \%)$ \\
\hline$\geqslant 90$ minutos/seção & $150(32,0 \%)$ & $205(35,9 \%)$ & $355(34,1 \%)$ \\
\hline \multicolumn{4}{|l|}{ Tempo de Prática } \\
\hline$\leqslant 3$ meses & $101(21,5 \%)$ & $87(15,2 \%)$ & $188(18,1 \%)$ \\
\hline $3-6$ meses & $92(19,6 \%)$ & $64(11,2 \%)$ & $156(15,0 \%)$ \\
\hline $6-12$ meses & $78(16,6 \%)$ & $96(16,8 \%)$ & $174(16,7 \%)$ \\
\hline $1-2$ anos & $97(20,6 \%)$ & $95(16,6 \%)$ & $192(18,6 \%)$ \\
\hline $2-5$ anos & $61(13,0 \%)$ & $115(20,1 \%)$ & $176(16,9 \%)$ \\
\hline$\geqslant 5$ anos & $41(8,7 \%)$ & $114(20,0 \%)$ & $155(14,9 \%)$ \\
\hline \multicolumn{4}{|l|}{ Objetivo Principal } \\
\hline Estético & $173(36,8 \%)$ & $129(22,5 \%)$ & $302(29,0 \%)$ \\
\hline Condicionamento Físico & $158(33,6 \%)$ & $273(47,8 \%)$ & $431(41,4 \%)$ \\
\hline Saúde & $122(25,9 \%)$ & $138(24,2 \%)$ & $260(25,0 \%)$ \\
\hline Lazer & $17(3,6 \%)$ & $31(5,5 \%)$ & $48(4,6 \%)$ \\
\hline
\end{tabular}

Com relação à adequação do primeiro subconjunto de dados para uso dos procedimentos da AFE, o valor do teste de KMO foi equivalente a 0,927 e o teste de esfericidade de Bartlett $\chi_{(298)}^{2}=5149,7(\mathrm{p}<0,001)$, apontando para a legitimidade da realização da análise fatorial. Informações disponibilizadas pela AFE podem ser observadas na Tabela 3. Mediante análise pormenorizada da matriz fatorial verifica-se que todos os valores de $\mathrm{r}$ associados ao peso fatorial apontaram significância estatística ( $\mathrm{p}<$ 0,001), não sendo encontrados itens com saturação fatorial $\geq 0,40$ em mais de um fator ou com saturação insuficiente. A solução fatorial da matriz de dados definiu seis fatores com eigenvalues superior a uma unidade, contribuindo para explicar conjuntamente por volta de $66 \%$ da variância total e comunalidades superiores a 0,46 . Definição de seis 
TABELA 2 - Estatística descritiva equivalente aos itens do Behavioral Regulation in Exercise Questionnaire (BREQ-3) traduzido e aplicado em adultos brasileiros de ambos os sexos $(n=1041)$.

\begin{tabular}{|c|c|c|c|c|}
\hline Item & Média & Desvio-Padrão & Assimetria & Curtose \\
\hline 1. É importante para mim fazer exercício físico regularmente & 2,79 & 1,09 & $-0,77$ & $-0,54$ \\
\hline 2. Não sei por que tenho que fazer exercício físico & 0,52 & 0,54 & 1,17 & 1,21 \\
\hline 3. Acredito que o exercício físico é divertido & 3,34 & 0,73 & $-0,63$ & 0,86 \\
\hline 4. Sinto-me culpado/a quando não faço exercício físico & 1,86 & 0,93 & 0,41 & $-0,82$ \\
\hline 5. Faço exercício físico porque é consistente com meus objetivos de vida & 2,75 & 0,93 & $-0,45$ & $-0,61$ \\
\hline 6. Porque outras pessoas dizem que devo fazer exercício físico & 1,19 & 0,90 & 1,05 & 0,89 \\
\hline 7. Valorizo os benefícios do exercício físico & 2,71 & 1,14 & $-0,65$ & $-0,79$ \\
\hline 8. Não vejo sentido em fazer exercício físico & 0,72 & 0,51 & 0,94 & 1,01 \\
\hline 9. Gosto das minhas sessões de exercício físico & 3,48 & 0,73 & 0,70 & 0,77 \\
\hline 10. Sinto-me envergonhado/a quanto falto a uma sessão de exercício físico & 1,73 & 0,84 & 0,68 & 0,75 \\
\hline 11. Considero que o exercício físico faz parte de mim & 3,24 & 0,81 & $-0,69$ & 0,16 \\
\hline 12. Meus amigos/familiares dizem que devo fazer exercício físico & 1,12 & 0,91 & 0,89 & 0,93 \\
\hline 13. Penso que é importante fazer algum esforço para praticar exercício físico & 2,49 & 1,23 & $-0,48$ & $-0,96$ \\
\hline 14. Não sei por que tenho que me incomodar em fazer exercício físico & 1,03 & 0,80 & 0,92 & 0,97 \\
\hline 15. Acho que o exercício físico é uma atividade prazerosa & 3,17 & 0,88 & $-0,94$ & $-0,16$ \\
\hline 16. Sinto-me fracassado/a quando não faço exercício físico por algum tempo & 1,76 & 0,91 & 0,52 & $-0,91$ \\
\hline 17. Vejo o exercício físico como parte fundamental do que sou & 3,22 & 0,98 & 0,55 & 0,87 \\
\hline 18. Outras pessoas vão ficar insatisfeitas comigo se não fizer exercício físico & 0,99 & 0,85 & 0,96 & 1,14 \\
\hline 19. Sinto-me ansioso/a se não fizer exercício físico regularmente & 2,38 & 1,25 & $-0,40$ & $-0,85$ \\
\hline 20. Acredito que fazer exercício físico é uma perda de tempo & 0,65 & 0,48 & 1,13 & 1,10 \\
\hline 21. Sinto-me bem e satisfeito quando faço exercício físico & 2,98 & 0,82 & $-0,12$ & $-0,90$ \\
\hline 22. Considero que o exercício físico esta de acordo com meus valores pessoais & 3,01 & 0,80 & $-0,40$ & $-0,55$ \\
\hline 23. Sinto-me pressionado pelos meus amigos/família para fazer exercício físico & 0,93 & 0,81 & $-0,90$ & 1,01 \\
\hline
\end{tabular}

fatores com igual quantidade e distribuição de itens em cada fator confirma proposta original do $B R E Q-3$, recomendando, desse modo, o uso de idênticas denominações: motivação intrínseca (fator 1), regulação integrada (fator 2), regulação identificada (fator 3), regulação introjetada (fator 4), regulação externa (fator 5) e amotivação (fator 6).

Com relação à magnitude dos índices de consistência interna dos seis fatores apontados pela estrutura fatorial, previamente aos calculados dos coeficientes alfa de Cronbach, foram conduzidas estatísticas preliminares que fundamentam suas estimativas - Tabela 4 . Os valores de média encontrados variaram de 0,83 a 3,22, com desvios-padrão entre 0,61 e 1,04. A princípio, esses achados referentes à estatística descritiva fundamenta fortemente a confiabilidade das estimativas de consistência interna, considerando que o valor médio de nenhuma das escalas, isoladamente, se aproximou dos escores extremos possíveis (0 ou 4). Destaca-se, ainda, que a variabilidade dos escores individuais foi restrita, denotando-se, portanto, alguma homogeneidade em sua dispersão, independente do fator considerado. Ao procederem os cálculos dos coeficientes alfa de Cronbach foram identificadas dimensões que variaram de 0,68 (REID) a 0,86 (MOTI), o que aponta para índices desejáveis de consistência interna para ambos os formatos da versão traduzida do BREQ-3. As correlações bivariadas inter-fatores apresentaram valores entre 0,11 e 0,75 .

Uma vez definida a estrutura fatorial mediante procedimentos da AFE, passou-se a analisar indicadores associados à validação do modelo proposto. Para tanto, foram empregados procedimentos da AFC no segundo subconjunto da amostra. Inicialmente, com auxílio do gráfico Box Plot, constatou-se a ausência de casos outliers, atendendo, desse modo, importante pressuposto para os procedimentos da AFC. 
TABELA 3 - Análise fatorial exploratória do Behavioral Regulation in Exercise Questionnaire (BREQ-3) traduzido e aplicado em adultos brasileiros de ambos os sexos.

\begin{tabular}{|c|c|c|c|c|c|c|}
\hline & Fator 1 & Fator 2 & Fator 3 & Fator 4 & Fator 5 & Fator 6 \\
\hline & MOTI & REIG & REID & REIJ & REEX & AMOT \\
\hline Item 3 & 0,83 & & & & & \\
\hline Item 9 & 0,79 & & & & & \\
\hline Item 15 & 0,85 & & & & & \\
\hline Item 21 & 0,83 & & & & & \\
\hline Item 5 & & 0,79 & & & & \\
\hline Item 11 & & 0,81 & & & & \\
\hline Item 17 & & 0,80 & & & & \\
\hline Item 22 & & 0,76 & & & & \\
\hline Item 1 & & & 0,68 & & & \\
\hline Item 7 & & & 0,85 & & & \\
\hline Item 13 & & & 0,77 & & & \\
\hline Item 19 & & & 0,78 & & & \\
\hline Item 4 & & & & 0,65 & & \\
\hline Item 10 & & & & 0,62 & & \\
\hline Item 16 & & & & 0,74 & & \\
\hline Item 6 & & & & & 0,69 & \\
\hline Item 12 & & & & & 0,72 & \\
\hline Item 18 & & & & & 0,78 & \\
\hline Item 23 & & & & & 0,70 & \\
\hline Item 2 & & & & & & 0,56 \\
\hline Item 8 & & & & & & 0,69 \\
\hline Item 14 & & & & & & 0,76 \\
\hline Item 20 & & & & & & 0,71 \\
\hline Eigenvalues & 12,51 & 5,07 & 4,12 & 3,48 & 2,89 & 1,79 \\
\hline \% Variação & 30,75 & 12,34 & 8,68 & 6,29 & 4,16 & 3,82 \\
\hline Variação Total & & 43,09 & 51,77 & 58,06 & 62,22 & 66,04 \\
\hline
\end{tabular}

MOTI: motivação intrínseca; REIG: motivação extrínseca de regulação integrada; REID: motivação extrínseca de regulação identificada; REIJ: motivação extrínseca de regulação introjetada; REEX: motivação extrínseca de regulação externa; AMOT: amotivação.

TABELA 4 - Estatística descritiva, coeficiente alfa de Cronbach e correlações bivariadas entre fatores do Behavioral Regulation in Exercise Questionnaire (BREQ-3) traduzido e aplicado em adultos brasileiros.

\begin{tabular}{lcccccccc}
\hline Fator & Média & Desvio-Padrão & Alfa de Cronbach & AMOT & REEX & REIJ & REID & REIG \\
\hline AMOT & 0,83 & 0,61 & 0,72 & & & & & \\
\hline REEX & 1,04 & 0,80 & 0,74 & 0,73 & & & & \\
\hline REIJ & 1,77 & 0,87 & 0,73 & 0,26 & 0,55 & & & \\
\hline REID & 2,62 & 1,04 & 0,68 & $-0,35$ & $-0,23$ & 0,36 & & \\
\hline REIG & 3,04 & 0,85 & 0,81 & $-0,55$ & $-0,28$ & 0,33 & 0,75 & \\
\hline MOTI & 3,22 & 0,79 & 0,86 & $-0,57$ & $-0,33$ & 0,11 & 0,71 & 0,73
\end{tabular}

AMOT: amotivação; REEX: motivação extrínseca de regulação externa; REIJ: motivação extrínseca de regulação introjetada; REID: motivação extrínseca de regulação identificada; REIG: motivação extrínseca de regulação integrada; MOṪ: motivação intrínseca.

Por intermédio da figura 2 visualizam-se informações equivalentes à estrutura fatorial do modelo proposto. De imediato, verifica-se que a AFC sustenta a hipótese da presença de seis fatores, conforme demonstrado pelos índices de ajuste equivalentes à $\chi^{2}=406,35, \mathrm{gl}=215, \chi^{2} / \mathrm{gl}=1,89, \mathrm{CFI}=0,931, \mathrm{GFI}=$ 
0,936, $\mathrm{AGFI}=0,955$ e RMSR $=0,067\left[\mathrm{IC}_{90 \%}: 0,064-0,071\right]$. Ainda, as cargas fatoriais oscilaram entre 0,65 e 0,86 acompanhadas de variâncias residuais satisfatórias, o que permite assumir, pelo viés da $\mathrm{AFC}$, a validade de construto da versão traduzida do $B R E Q-3$ para uso em adultos brasileiros.

Os valores associados à VEM dos fatores de primeira ordem do modelo proposto oscilaram entre 0,64 (MOTI) e 0,59 (AMOT), enquanto as dimensões equivalentes à CC variaram entre 0,74 (AMOT) e 0,83 (REIG), suportando fortemente a validade convergente da solução fatorial ajustada para a versão do $B R E Q-3$ tratada no estudo.

Indicadores relacionados aos testes de invariância fatorial entre diferentes estratos associados ao sexo e à idade são disponibilizados na Tabela 5. A análise multigrupo conduzida para sexo apontou valores de $\Delta \chi^{2}$ e $\Delta$ CFI que revelam a existência de invariância entre mulheres e homens na estrutural fatorial do modelo considerado. De igual modo, encontraram-se indicadores que sugerem bons ajustes para os modelos que fixaram cargas fatoriais, variância/covariâncias e resíduos nos três grupos de idade ( $\leq 20$ anos, 21-40 anos e $\geq 41$ anos). Contudo, salienta-se que, mesmo se apresentando adequados, os ajustes foram mais aprimorados quando se comparou os estratos relacionados ao sexo que quando se comparou os estratos relacionados à idade.

TABELA 5 - Indicadores produzidos pela análise fatorial confirmatória multigrupo para testes de invariância fatorial entre diferentes estratos relacionados ao sexo e à idade.

\begin{tabular}{|c|c|c|c|c|c|c|c|}
\hline & $x^{2}$ & $\mathrm{gl}$ & $\Delta \mathrm{x}^{2}$ & $\Delta \mathrm{gl}$ & valor $p$ & $\mathrm{CFI}$ & $\triangle \mathrm{CFI}$ \\
\hline \multicolumn{8}{|l|}{ Sexo } \\
\hline Modelo 1 & 652,60 & 430 & & & & 0,925 & \\
\hline Modelo 2 & 661,68 & 444 & 9,08 & 14 & $>0,05$ & 0,924 & 0,001 \\
\hline Modelo 3 & 667,13 & 446 & 14,53 & 16 & $>0,05$ & 0,922 & 0,003 \\
\hline Modelo 4 & 676,52 & 458 & 23,92 & 28 & $>0,05$ & 0,919 & 0,006 \\
\hline \multicolumn{8}{|l|}{ Idade } \\
\hline Modelo 1 & 665,43 & 430 & & & & 0,920 & \\
\hline Modelo 2 & 676,67 & 444 & 11,24 & 14 & $>0,05$ & 0,913 & 0,007 \\
\hline Modelo 3 & 685,29 & 446 & 19,86 & 16 & $>0,05$ & 0,911 & 0,009 \\
\hline Modelo 4 & 695,48 & 458 & 30,05 & 28 & $>0,05$ & 0,908 & 0,012 \\
\hline
\end{tabular}

Modelo 1: Modelo de configuração (todos parâmetros estão livres para serem estimados); Modelo 2: Modelo em que cargas fatoriais são contrastadas; Modelo 3: Modelo em que variância/covariâncias são contrastadas; Modelo 4: Modelo em que resíduos são contrastados.

\section{DISCUSSÃO}

A efetivação das etapas do processo de tradução do instrumento não apresentou maior dificuldade devido à metodologia adotada e à estrutura simples e objetiva de formulação dos itens do $B R E Q-3$. A tradução inicial realizada pelos dois tradutores foi pouco modificada nas etapas subsequentes. A retrotradução, quando comparada ao instrumento original, apresentou discretas discrepâncias, resultantes de ajustes realizados para atender especificidades de determinados itens. A análise das equivalências semântica, idiomática, cultural e conceitual, equivalente à adaptação transcultural, como a etapa de tradução, indicou que o instrumento foi de fácil tradução.

A análise das equivalências mostrou que os domínios do BREQ-3 são apropriados e os atributos utilizados na versão original do instrumento são igualmente válidos para a cultura-alvo, o que atende a equivalência cultural. A 


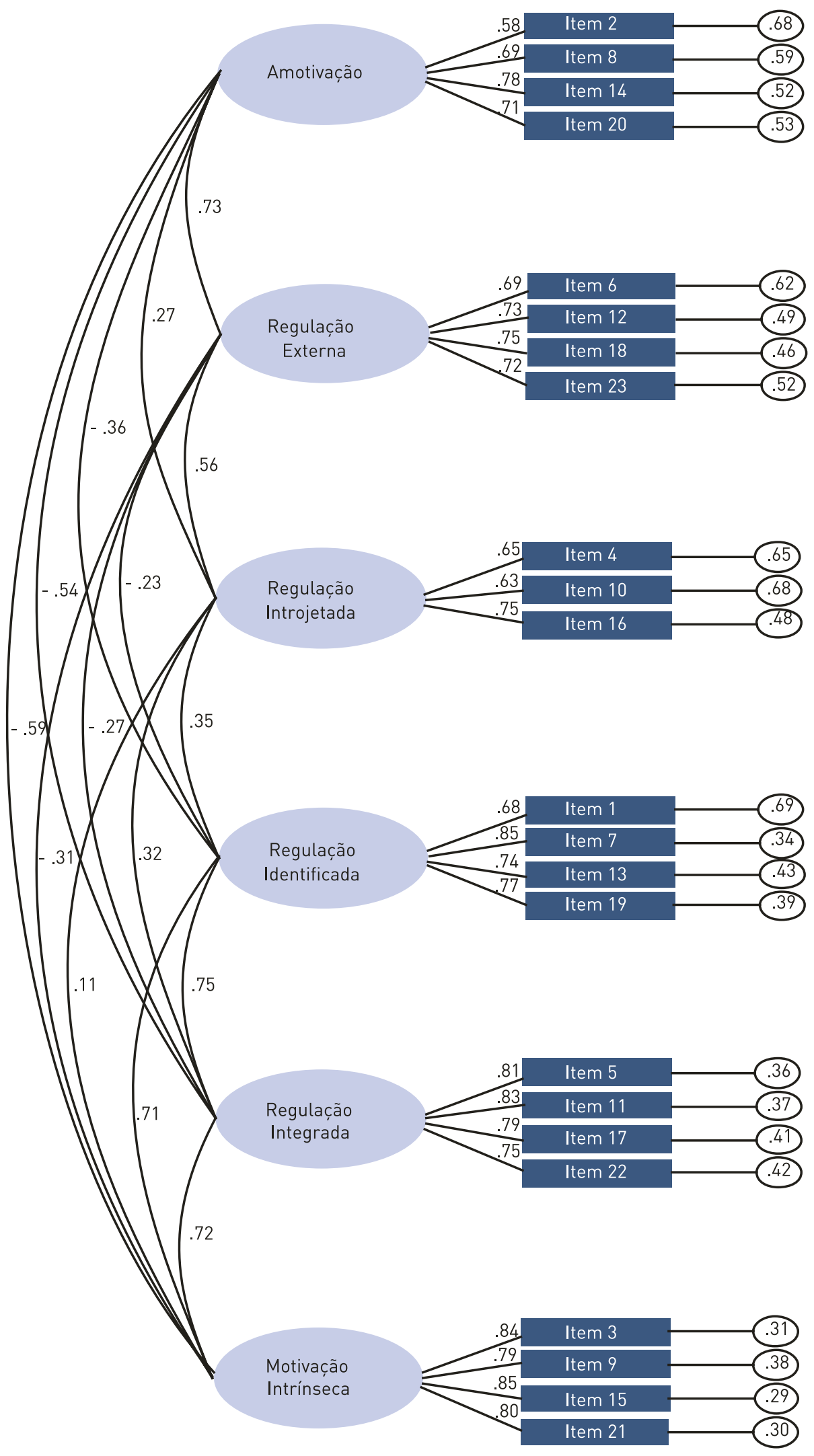

FIGURA 2 - Estrutura fatorial do Behavioral Regulation in Exercise Questionnaire (BREQ-3) traduzido e aplicado em adultos brasileiros. As elipses representam as escalas e os retângulos os itens do questionário. As variâncias residuais são mostradas nos círculos menores. 
equivalência conceitual indicou que poucos itens necessitaram de ajustes. Os itens puderam ser considerados de maneira semelhante ao formato original, indicando, mais uma vez, que a estrutura de formulação do $B R E Q-3$ foi bem elaborada. No que se refere à equivalência idiomática, a versão traduzida mostrou que quase a totalidade dos itens foram avaliados como "inalterado" e nenhum membro do comitê de análise considerou algum item como "muito alterado" ou "completamente alterado", quando da comparação entre as versões dos instrumentos original, traduzido e retrotraduzido.

Quanto à estrutura fatorial do $B R E Q-3$ traduzido e adaptado no presente estudo para o idioma português, constatou-se disposição semelhante a encontrada na versão original proposta por Wilson et al. ${ }^{15}$, sendo extraída idêntica quantidade de fatores equivalentes à motivação. Ainda, com exceção da REID que apresentou valor equivalente ao alfa de Cronbach de 0,68, os demais fatores de motivação extraídos da estrutura fatorial apresentaram consistência interna superior a 0,70. Considerando que o fator associado à REID é o único do instrumento com três itens, assume-se que sua consistência interna pode ser marginalmente considerada. Portanto, existem indicações de que a versão traduzida do $B R E Q-3$ apresenta critérios de aceitabilidade quanto à consistência interna, o que aponta sua confiabilidade para analise do perfil de motivação em adultos praticantes de exercício físico no contexto brasileiro.

Porém, observou-se que, em comparação com a versão original, a consistência interna de cada escala de motivação, de modo geral, foi discretamente mais baixa na estrutura fatorial do $B R E Q-3$ traduzido para o idioma português. Também, a amplitude de variação entre os escores mais elevado $(0,86)$ e mais baixo $(0,68)$ foi superior a apresentada pela versão original do $B R E Q-3$ $(0,86$ e 0,73 , respectivamente), o que sugere menor equilíbrio entre os fatores de motivação na versão traduzida para o idioma português.

Provável justificativa para esses achados possam estar associada às características das amostras selecionadas em um e outro estudo. Originalmente, o $B R S Q-3$ foi aplicado e validado em uma amostra de universitários do Canadá, com idade média próxima de 20 anos, enquanto no presente estudo foram reunidos sujeitos frequentadores de Clube de Fitness com idades entre 18 e 54 anos. Portanto, é possível que os contextos em que ambos os estudos foram realizados possam ter definido diferenças quanto ao perfil de interesse e motivos para a prática de exercício físico.

Outra opção de análise da validade dos fatores teóricos que compõem o $B R S Q-3$ traduzido para o idioma português é mediante as dimensões dos coeficientes de correlação inter-fatores decorrentes da definição dos construtos, como forma de complemento da análise fatorial, uma vez que, em teoria, devem se portar de acordo com o continuum de autodeterminação. Neste caso, constata-se que a disposição dos valores de $r$ entre os fatores observados na estrutura fatorial confirmam a presença do continuum de autodeterminação na versão traduzida do $B R S Q-3$, considerando que regulações próximas uma das outras no continuum mostraram estar fortemente correlacionadas em um sentido positivo, quando comparadas com regulações mais afastadas no continuum. Fato similar também foi relatado no estudo original de proposição do $B R S Q$ 3, o que reforça a tese de que este instrumento se define como ferramenta de análise das regulações de motivação de praticantes de esporte sob a luz da TaD.

Em tese, a análise da invariância fatorial de um instrumento permite verificar 
a possibilidade dos dados disponibilizados por sua escala de medida identificar o construto em questão de maneira similar nos diferentes substratos da amostra de validação, minimizando, dessa forma, que eventuais diferenças observadas entre os estratos possam ser atribuídas às inconsistências de suas propriedades psicométricas. Neste caso, outro importante achado do presente estudo foi a confirmação da invariância fatorial para sexo e idade, demonstrando que existem fortes indícios no sentido de que a versão tratada do $B R E Q-3$ possa identificar de forma equivalente as regulações motivacionais para a pratica de exercício físico em mulheres e homens, independentemente da idade entre 18 e 60 anos $^{30}$.

Entre as possíveis limitações derivadas da aplicação do $B R S Q-3$ como instrumento de coleta de dados, está a veracidade das respostas com que os participantes do estudo se posicionaram em relação à prática de exercício físico, considerando que as informações apresentadas no questionário são auto-relatadas. No entanto, auto-relato é o procedimento corrente em levantamentos com essas características, sendo a forma mais viável de reunir dados em estudos com este propósito. $\mathrm{O}$ fato de alguns itens, individualmente, apresentar discreto desvio da distribuição normal, também deve ser considerado. Outra limitação do estudo consiste na situação de que, mesmo que a amostra tenha reunido grande quantidade de sujeitos ( $\mathrm{n}=1041)$, a seleção não foi aleatória. Portanto, pode não ser verdadeiramente representativa da população delimitada.

Concluindo, a versão do instrumento $B R S Q-3$ traduzida e adaptada para o idioma português alcançou bom desempenho psicométrico frente à amostra do presente estudo, apresentando satisfatórios coeficientes alfa de Cronbach calculados para os fatores de motivação gerados. A solução fatorial encontrada mediante a AFE, e validada por intermédio de indicadores produzidos pela $\mathrm{AFC}$, foi similar à apresentada originalmente com confirmação da invariância fatorial para sexo e idade. Desta maneira, a versão do $B R S Q-3$ disponibilizada no presente estudo mostra-se promissora para utilização em futuras intervenções com objetivo de analisar as regulações de motivação para a prática de exercício físico de adultos brasileiros.

\section{REFERÊNCIAS}

1. Reiner M, Niermann C, Jekauc D, Woll A. Long-term health benefits of physical activity - a systematic review of longitudinal studies. BMC Public Health. 2013. 13:813.

2. Varo JJ, Martínez-Gonzáles MA, Irala-Estévez J, Kearney J, Gibney M, Martínez JA. Distribution and determinants of sedentary lifestyles in the European Union. International Journal of Epidemioly. 2003. 32:138-46.

3. Ferreira MS, Najar AL. Programas e campanhas de promoção da atividade física. Ciência \& Saúde Coletiva. 2005. 10:207-19.

4. Anderson CB. When more is better: number of motives and reasons for quitting as correlates of physical activity in women. Health Education Research. 2003. 18:525-37.

5. Ebben W, Brudzynski L. Motivations and barriers to exercise among college students. Journal of Exercise Physiology Online. 2008. 11(5):1-11.

6. Gill DL, Williams L. Psychological Dynamics of Sport and Exercise. $3^{\text {a }}$ Edition. Champaign, Illinois: Human Kinetics. 2008.

7. Deci EL, Ryan RM. Intrinsic motivation and self-determination in human behavior. New York: Plenum, 1985.

8. Ryan RM, Deci EL. Self-determination theory and the facilitation of intrinsic motivation, social development, and well-being. American Psychologist. 2000. 55:68-78.

9. Vallerand R. Intrinsic and extrinsic motivation in sport and physical activity: a review and a look at the future. In: Tennenbaum G, Eklund R (Eds.). Handbook of Sport Psychology. 3rd Edition. New York: John Wiley \& Sons. 2007. pp. 59-83. 
10. Hagger MS, Chatzisarantis NLD. Integrating the theory of planned behavior and selfdetermination theory in health behavior: a meta-analysis. British Journal of Health Psychology. 2009. 14:275-302.

11. Chiskvo VI. A cross-cultural analysis of autonomy in education: a self-determination theory perspective. Theory and Research in Education. 2009. 7:253-262.

12. Li F. The Exercise Motivation Scale: its multifaceted structure and construct validity. Journal of Applied Sport Psychology. 1999. 11:97-115.

13. Mullen E, Markland D \& Ingledew DK. A graded conceptualization of selfdetermination in the regulation of exercise behavior: development of a measure using confirmatory factor analysis. Personality and Individual Differences. 1997. 23:745-752.

14. Markland D, Tobin V.A modification to behavioural regulation in exercise questionnaire to include an assessment of amotivation. Journal of Sport and Exercise Psychology. 2004. 26:191-196.

15. Wilson PM, Rodgers WM, Loitz CC \& Scime G. "It's who I am .... Really!". The importance of integrated regulation in exercise contexts. Journal of Applied Biobehavioral Research. 2006. 11:79-104.

16. Teixeira PJ, Carraça EV, Markland D, Silva MN, Ryan RM. Exercise, physical activity and self-determination theory: a systematic review. International Journal of Behavioral Nutrition and Physical Activity. 2012. 9:78.

17. Moreno JA, Cervelló EM, Martínez A. Measuring self-determination motivation in a physical fitness setting: validation of the Behavioural Regulation in Exercise Questionnaire-2 in a Spanish sample. Journal of Sport Medicine and Physical Fitness. 2007. 47(3):366-78.

18. Palmeira A, Teixeira P, Silva M, Markland D. Confirmatory Factor Analysis of the Behavioural Regulation in Exercise Questionnaire - Portuguese Version. 12th European Congress of Sport Psychology, Halkidiki, Greece, 4-9 September, 2007.

19. Moustaka FC, Vlachopoulos SP, Vazou S, Kaperoni M., Markland D. Initial validity evidence for the Behavioural Regulation in Exercise Questionnaire - 2 among Greek exercise participants, European Journal of Psychological Assessment. 2010. 26(4): 269-76.

20. Farmanbar R, Niknami S, Hidarnia A, Lubans DR. Psychometric Properties of the Iranian Version of the Behavioral Regulation in Exercise Questionnaire-2 (BREQ-2). Health Promotion Perspectives. 2011. 1(2):95-104.

21. Rutten GM, Meis JJ, Hendriks MR, Hamers FJ, Veenhof C, Kremers SP. The contribution of lifestyle coaching of overweight patients in primary care to more autonomous motivation for physical activity and healthy dietary behaviour: results of a longitudinal study. International Journal of Behavioral Nutrition and Physical Activity.2014. 11:86.

22. Craike MJ, Polman R, Eime R, Symons C, Harvey J, Payne W. Associations between behavior regulation, competence, physical activity, and health for adolescent females. Journal of Physical Activity and Health. 2014. 11(2):410-8.

23. Sibley BA, Hancock L, Bergman SM. University students exercise behavioral regulation, motives, and physical fitness. Perceptual Motor Skills. 2013. 116(1): 322-39.

24. Cid L, Moutão J, Leitão J, Alves J. Behavioral regulation assessment in exercise: exploring an autonomous and controlled motivation index. Spanish Journal of Psychology. 2012. 15(3):1520-8.

25. Viana MS. Motivação de adolescentes para a prática de exercícios físicos: perspectivas da teoria da autodeterminação. Dissertação de Mestrado. Universidade do Estado de Santa Catarina. Florianópolis: UDESC. 2009.

26. Hair J, Black W, Babin B, Anderson R, Tatham R. Multivariate Data Analysis. 6th Edition. New Jersey: Pearson Educational, Inc, 2006.

27. Hambleton RK. Issues, design and technical guidelines fot adapting tests into multiple languages and cultures. In: Hambleton RK, Merenda PF, Spielberger CD (Eds). Adapting Psychological and Educational Tests for Cross-Cultural Assessment. Mahwah, NJ: Lawrence Erlbaum. 2005. pp. 3-38.

28. ABEP - Associação Brasileira de Empresas de Pesquisa. Critério de Classifficação Econômica Brasil. São Paulo: Associação Brasileira de Empresas de Pesquisa, 2009.

29. Hu L, Bentler P. Cutoff criteria for fit indexes in covariance structure analysis: conventional criteria versus new alternatives. Structural Equation Modeling. 1999. 6(1):1-55.

30. Byrne BM. Structural Equation Modeling with AMOS: Basic Concepts, Applications, and Programming. Mahwah, New Jersey: Lawrence Erlbaum Associates, Inc., 2010. 